MODELING, IDENTIFICATION AND CONTROL, 1999, vOL. 19, NO. 4, 207-223

doi:10.4173/mic.1998.4.3

\title{
The macroeconomy as a network of money-flow transfer functions
}

\author{
TROND ANDRESEN†
}

Keywords: Macroeconomics, network, block diagram, time lag, simulation

An introduction on A. W. Phillips "hydraulic" macroeconomic models is given. His (and others economists') notion that a macroeconomy may reasonably be considered to have dynamics corresponding to a first order time lag transfer function, is justified in this paper by aggregation of individual micro agents. In connection with this economic application, we derive and discuss a theorem and some rules for general networks of time lagged blocks. Finally, Monte Carlo simulations of networks of micro agents are undertaken, supporting the validity of the first order time lag aggregate model.

\section{Introduction}

A macroeconomy evolves through time, and may be viewed as a collection of sub-entities interacting with each other. It is thus a dynamic system. Dynamics are mathematically and conceptually much more complicated than (comparative) statics. When doing dynamics, algebraic equations (corresponding to the intersecting schedules widely used in economics) are substituted with differential or (in the discrete time case, difference) cquations. These equations are difficult to work with in the sense that one can hardly - as one can in a static framework-find graphic or algebraic (when possible) solutions to them without computer-implemented solution software. Furthermore, it is very difficult to gain any qualitative insights about the behaviour of a dynamic system by inspecting its differential equations. The method of representing the system graphically through block diagrams lends itself much easier to such insights. This way of representing a system may be considered an interface between the user and the differential equation based model.

In two seminal papers (Phillips 1954, 1957), A. W. Phillips (who today is known in economics almost exclusively for the Phillips curve) did the above' ${ }^{\prime}$. He modeled the macroeconomy as a dynamic system consisting of interconnected sub-entities. In the first paper he found the algebraic solutions to the models, while in connection with the latter paper he had access to an analog computer for numerical simulation. At the time, this was pioneering work. It was a second stage after his initial and simpler physical hydraulic simulation model with vessels interconnected by tubes. Phillips had the advantage of a background as an electrical engineer, and acquainted himself with the fairly new discipline of control engineering and theory that had evolved strongly during the Second World War. He saw that it could be applied to economics.

After an initially enthusiastic reception, however, this research was subsequently disparaged by many in the economics profession as "hydraulic Keynesianism". This was possible not the least since at that time very few economists had access to, or were

†Department of Engineering Cybernetics, The Norwegian University of Science and Technology, N-7034 Trondheim, Norway. E-mail: Trond.Andresen@itk.ntnu.no.

'I am indebted to Professor P. N. (Raja) Junankar, who pointed out these articles to me, after observing similarities with my own work. 


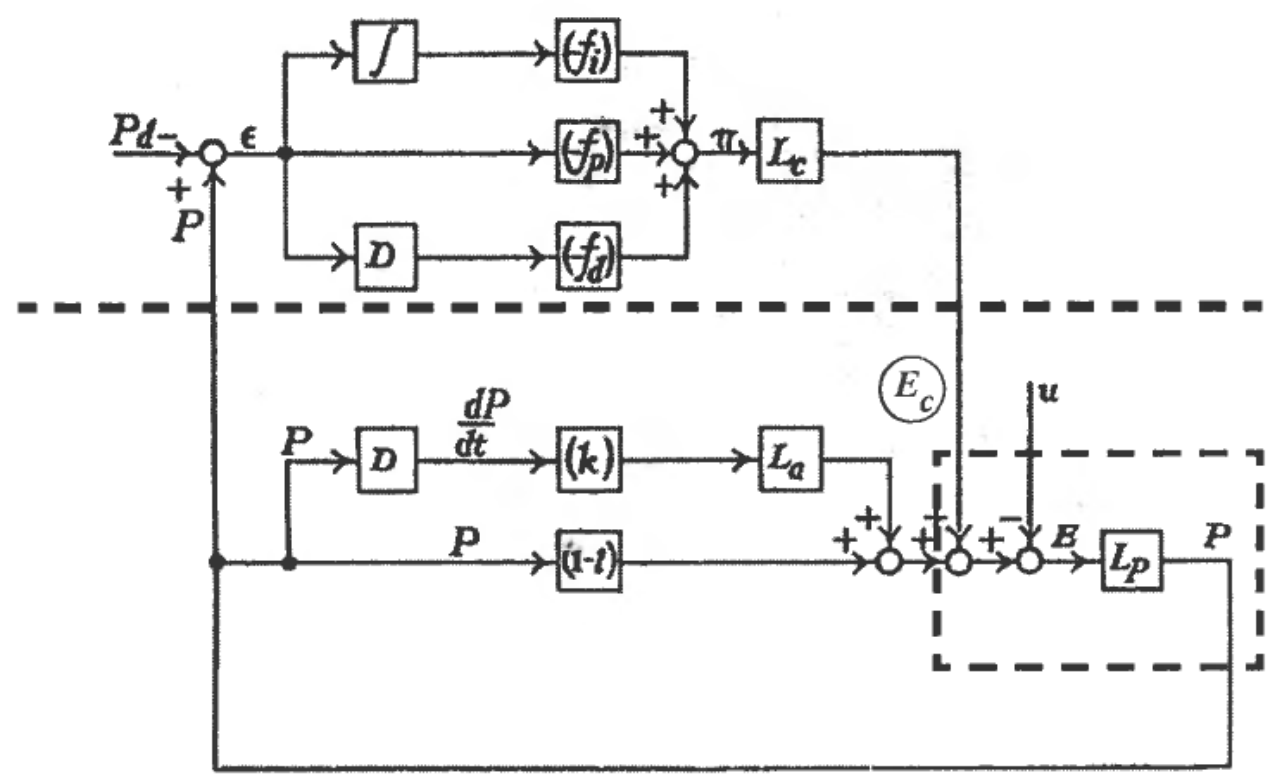

Figure 1. Facsimile of original Phillips block diagram.

acquainted with, the methods and the few and expensive tools available for simulating dynamic models.

Figure 1 shows a facsimile from Phillips' 1954 paper, the simplest model with fixed prices. The thick dotted line is added here. Above this line is the control system. It compares actual output $P$ to desired output $P_{d}$, and the error $\varepsilon$ is fed into a PID controller. Note that these variables are money flows [currency unit/time unit] since the model is in continuous time, as opposed to what is usual in dynamic economics, where the time axis is partitioned into periods, and variables therefore are money amounts. The PID controller decides the intervention strategy of the government. The control action is government spending, indicated by the symbol $E_{c}$ (I have put a circle around the symbol to indicate that it is inserted here and not part of Phillips' original figure). It is not the purpose of this paper to discuss the control strategy suggested by Phillips, but only his model of the demand-to-output relationship, equation (1) below. The dotted rectangle (inserted by me) indicates this part of his model, a "vessel" fed by the incoming aggregate demand flow $E$. The block is called $L_{p}$ in Phillips' notation, and is a first order time lag,

$$
\frac{P}{E_{C}}(s)=h(s)=\frac{1}{1+T_{p} s}
$$

The rationale for the model (1) has traditionally been explained like this: The economy needs time to adjust to a change in demand. The first order time lag is the simplest model for such dynamics. Thus Phillips and others (for instance Godley and Cripps, 1983) use an “Occam's razor" type of justification for their choice of model.

The time lag model corresponds well (at least as a linearized approximation) to a vessel. A sudden increase in the incoming flow will initially increase the level of fluid 


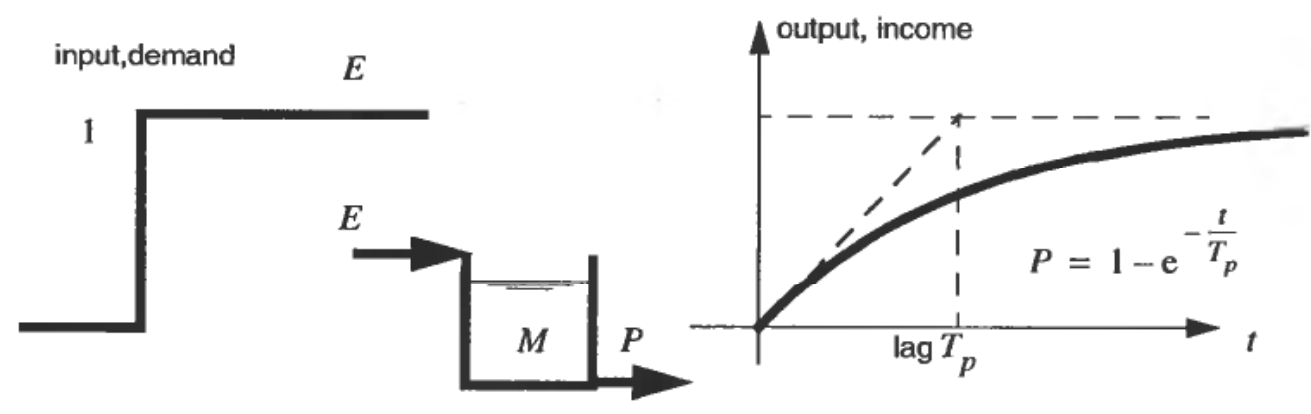

Figure 2. First-order time-lagged response.

(in our case: money), which leads to increased outflow in the next round. This is portrayed in Figure 2.

The economy is assumed to react to a jump of one unit in the demand flow with a time-dispersed exponential output response asymptotically approaching the incoming flow level. When the output flow $\boldsymbol{P}$ (theoretically) has reached that asymptotic level, we have equilibrium. $T_{p}$ is the time lag describing the speed of adjustment.

This paper will strengthen the validity of the time lag model by deriving it from the fact that the economy is an aggregate of a large number of individual agents (firms, households or both together). Our approach may introductorily be explained as follows: Assume a jump in demand. The increased flow of money percolates through the interconnected network of tens of thousands of firms that constitute the demand-to-output part of the economy, and gradually (but not immediately) the effect will show up as increased income for the factors of production (i.e. output). The lag for the aggregate is a consequence of $t w o$ different factors: The time lag of each firm on the micro level, and the degree to which the average unit of money flows to many other firms before it leaves the aggregate as income to the factors of production.

Money stock $M$ must be the integrated difference between demand- and output-flows. We have the stock/flow balance equation

$$
\dot{M}(t)=-P(t)+E(t)
$$

At the same time we want a step response as in Figure 2, corresponding to the transfer function (1). If we choose

$$
P(t)=M(t) / T_{p},
$$

this is satisfied. Equation (3) is intuitively appealing in the sense that the outgoing flow is proportional to money stock, which can be regarded (by the physical "vessel" analogy) as a "pressure" driving this flow. And the larger the time lag $T_{p}$, the less flow $P$ for a given $M$, i.e. a large time lag means that money has to accumulate significantly before it leads to increased spending.

In Phillips' model in Figure 1, we note that time lags also show up in two other places in his diagram, labeled $L_{c}$ and $L_{a}$ : The first is a lag in the government's (control action) spending, which may be interpreted as either sluggishness in ascertaining the current economic situation, sluggishness in implementing the intervention policy, or a combination of both. The second lag accounts for a sluggishness in the investment spending reaction of investors to the rate of change in output ( $D$ is a differentiation operator in the block diagram), through an "accelerator" coefficient.

So far on the Phillips model. The interested reader is referred to the original papers. 


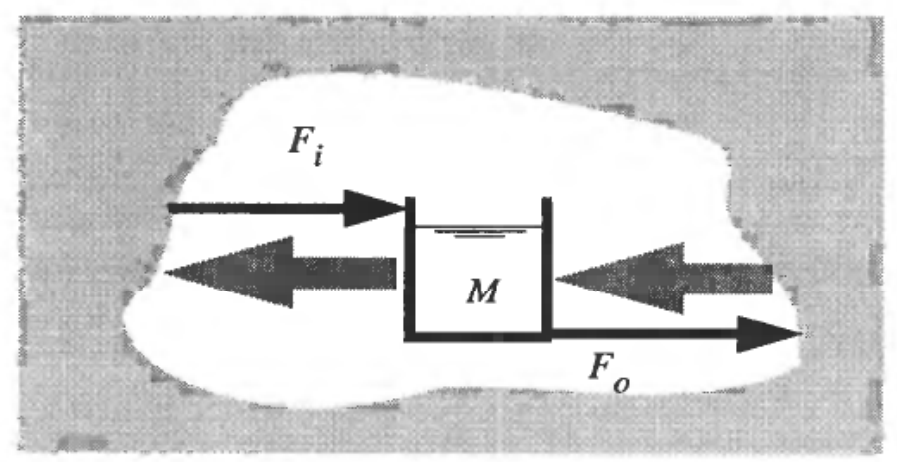

Figure 3. A generic microeconomic agent.

\section{From micro to macro agents}

We now choose the first-order time lag model as a candidate description of the behaviour of an individual "micro" agent; a household, a firm, a bank, a government. This generic economic agent concept is indicated in Figure 3.

The agent is again compared to a vessel with varying volume of fluid. Income money flow $F_{i}$ and spending flow $F_{o}$ are shown as black arrows. Real flows (labour, goods, services) are suggested by the thick shaded arrows in the Figure. The grey shaded area surrounding the agent is simply the set of all other agents, i.e. the macroeconomic system.

Money stock $M$ for the agent is the volume in the vessel at a given instant. $M$ may be interpreted as the agent's necessary liquid buffer to handle discrepancies between in- and outgoing money flows. This buffer is needed since both income and spending - seen from the individual agent-will fluctuate in a more or less unpredictable manner. This uncertainty is the only rationale for an agent to hold money, as opposed to non-liquid return-yielding financial assets.

Money stock may also be interpreted as due to a necessary "decision + action time delay" $\tau$ for the agent before received money is passed on again. We may think of this time delay in terms of a specific "packet" of money arriving at the inlet, appearing at the outlet $\tau$ time units later. For the special case with $F_{o}=F_{i}=F$ constant, $M$ will also be constant. We have

$$
M=F \tau, \text { or } \tau=M / F
$$

From (4) follows that a local velocity of money is:

$$
v=1 / \tau
$$

The delay associated with flows in general (as in process plants, pipelines, etc.), will in the case of money be the time a given amount spends between arrival and departure at a given agent. Flows between agents may be reckoned as immediate. Thus money always resides at some agent.

The agent is, just as in the aggregate case, assumed to react to a monetary step function income flow with a time-dispersed exponential spending response asymptotically approaching the incoming flow level, as already depicted in Figure 2. 
(no

income)

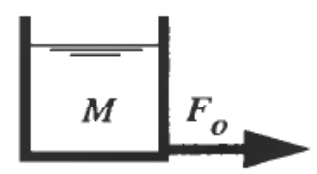

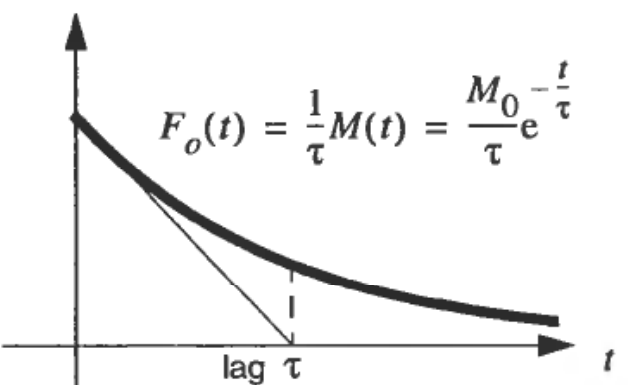

Figure 4. Time path for a micro agent with money but no income.

As in the aggregate case, we have

$$
F_{o}(t)=\frac{1}{\tau} M(t)
$$

The larger the time lag $\tau$, the less flow $F_{o}$ for a given $M$, i.e. a large time lag (lower money velocity $1 / \tau$ ) means that money has to accumulate significantly at the agent before the agent increases spending. The parameter $\tau$ is our first behavioural assumption for our generic agent. One may let $\tau$ be influenced by other system variables, for instance let it increase sharply due to mood changes in a recession/depression ( $\tau$ is a measure of liquidity preference, sec corollary 1.4 further below) or decrease with increasing interest rates. Such modifications will make a model consisting of several such agents, nonlinear. But for the time being we will stick to the assumption of a constant and identical $\tau$ for all agents.

We have considered the dynamics of an agent with initial zero money stock and a constant inflow of money starting at $t=0$. If we alternatively consider a situation with a certain initial money stock $M_{0}$ but no income, i.c. $F_{i}(t)=0$, then our agent, following (1) and (6), spends her money following a decaying exponential curve, which seems quite reasonable in a situation with zero income. See Figure 4.

\subsection{An aggregation theorem}

Let us now return to the aggregate, and let it consist of a large number of individual agents as described above. An aggregate "agent" (a sector) may for instance represent all firms, as in Phillips' macroeconomic model. The individual agents that constitute a given sector will of course have different "sizes" in the sense that money stock and flow magnitudes will vary widely between them. But we assume that (6) holds for all agents in a given aggregate, i.e. that the spending flow from an agent is proportional to the agent's money stock, by a common constant velocity factor $1 / \tau$ (this assumption will be relaxed later on). Thus all agents in a given sector is represented by a transfer function of the type ${ }^{2}$

\footnotetext{
${ }^{2}$ Here $s$ is a differentiation operator, so that
}

$$
y(t)=\frac{1}{1+\tau s} x(t) \Leftrightarrow(1+\tau s) y(t)=x(t)
$$

shall be interpreted as

$$
\frac{d y}{d t}=\frac{1}{\tau}(-y(t)+x(t))
$$

i.e. a linear differential equation with input $x$ and output $y$. 


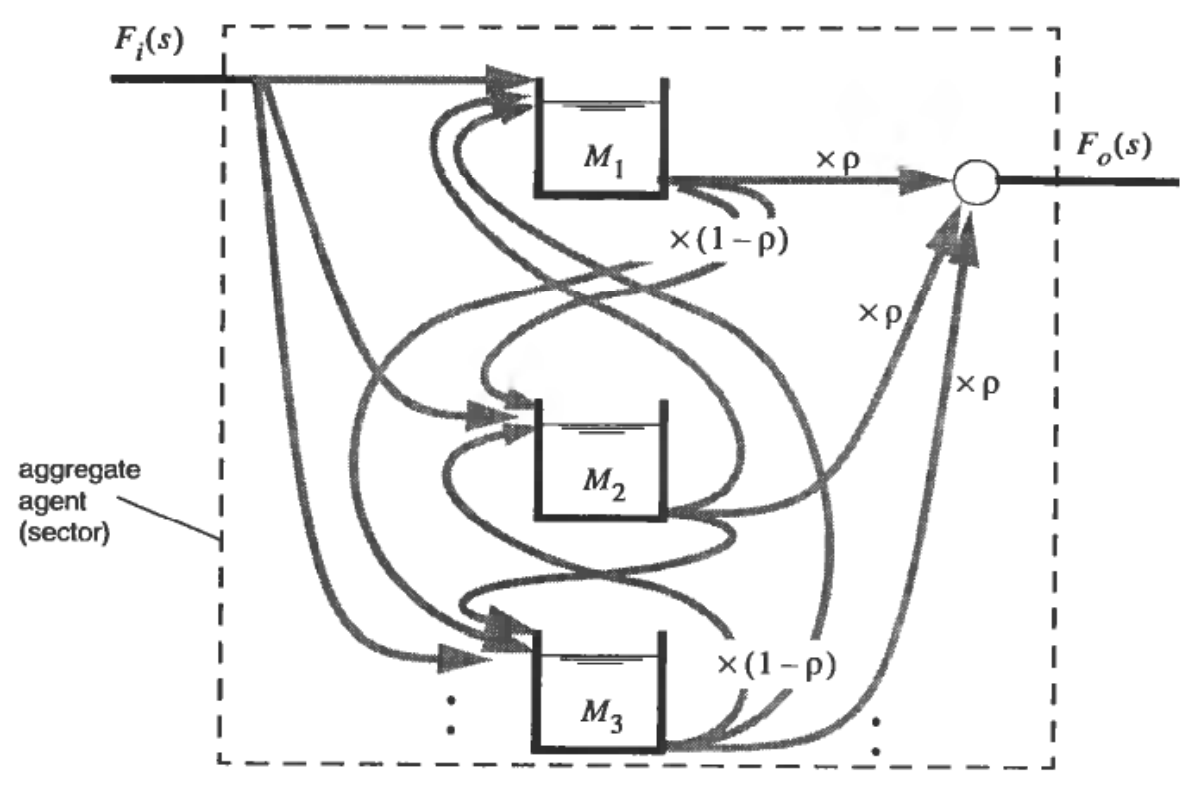

Figure 5. A flow network of "vessel" agents.

$$
h(s)=\frac{1}{1+\tau s}
$$

We furthermore assume that any (in an average sense) individual agent's outgoing money flow is divided into a share $\rho$ (out of the sector) and $(1-\rho)$ (to other agents within the sector), where $0<\rho \leq 1$. We call $\rho$ an outside spending coefficient. See Figure 5. The shaded arrows indicate a network of interactions, where any individual agent in principle interacts with any other agent. Our interest is focused on two aspects, input-output characteristics of the aggregate, and the dynamics of aggregate money stock.

Under the above assumptions the transfer function for the sector turns out to be surprisingly simple. It is given by the following "network aggregation theorem":

Theorem 1: Given a network of an infinite number of identical blocks which are first order transfer functions of the type (7), and which are interconnected by arbitrary coefficients, such that all transfer functions have identical outside spending coefficients $\rho$, and such that the remaining output coefficients for each transfer function sum to $1-\rho$. Then the transfer function for the network, between any block input and any block output, is

$$
h_{a}(s)=\frac{1}{1+T_{a} s}, \text { where } T_{a}=\frac{\tau}{\rho}
$$

Before proceeding with the proof, some comments to indicate that this result is intuitively satisfying. Let us first consider a type of sector where the population of agents have a low volume of monetary transactions between them, even if the number of agents may be large: A case in point is the aggregate of all households. In this case $\rho$ is close to unity. Referring to Figure 5, this means that the agents are simply laid out "in parallel", with negligible flows between them. Money arriving at a specific agent will emerge from the agent and also the household sector, without having to "percolate" via 
other household agents first. People use most of their income for purchasing goods and services from firms, not paying it to other households. Thus one should expect the aggregate of households to have the same fast response as an individual agent. This also fits with (8), since $T_{a}=\tau$ in the limit when $\rho=1$.

The other extreme is when the "aggregate agent" is such that agents mostly do their transactions with other agents within the aggregate. This case fits well with what financial sectors have developed into for the last decade. An outside agent who injected money into such an aggregate, would-if she had the means to trace that packet of money-observe that it would take a very long time before the last residue of the injected amount emerged from the aggregate. This case corresponds to $\rho$ being close to zero. It is consistent with (8), where a small $\rho$ means a large lag $T_{a}$, giving just the type of low-amplitude, drawn-out response that seems reasonable.

We will now prove Theorem 1.

Proof: In deriving the transfer function for the aggregate agent, we may assume that the outside incoming monetary flow arrives at one agent only, because of the symmetry between the agents, and because of the superposition principle that applies to a linear system: If the incoming flow was instead distributed between several agents, the resulting response would be the sum of responses to each component of the incoming flow, transmitted through identical transfer functions, which would then sum up to the same result we get when the incoming flow is assumed to arrive at a single agent only.

Consider the structure in Figure 6.

This block diagram accounts for the way an incoming monetary flow branches through the aggregatc of agents. As already argued we may assume that the flow enters at one single agent, in Figure 6 chosen as the uppermost. This results in a spending flow which is partioned into a share $\rho$ leaving the aggregate, and a share $1-\rho$ to another identical agent within the aggregate. The latter flow again results in a flow that is partioned into a share $\rho$ leaving the aggregate, and a share $1-\rho$ to another agent within the aggregate, and so on.

Note that feedback loops are indirectly accounted for by the structure in Figure 6, since the effect of any feedback loop may be equivalently represented by an infinite

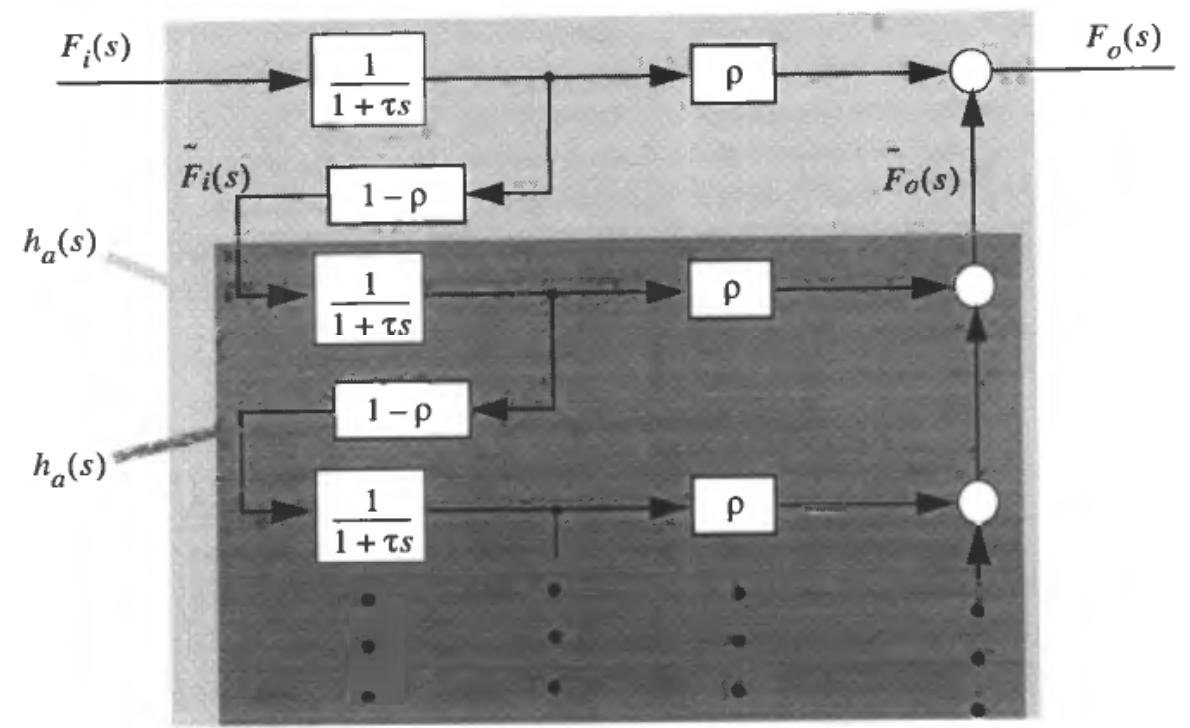

Figure 6. An input-output equivalent network that is without feedback loops. 
succession of series and parallel connections through identical transfer functions. The transfer function for the aggregate,

$$
h_{a}(s)=F_{o}(s) / F_{i}(s)
$$

is indicated in Figure 6 by the light shaded area.

If we now remove the upper single agent from the aggregate, and assume that the remaining number of agents is so large that this does not significantly affect the dynamics of the aggregate, then $h_{a}(s)$ may also be found as indicated by the dark shaded area,

$$
h_{a}(s)=\tilde{F}_{o}(s) / \tilde{F}_{i}(s)
$$

Employing rules for manipulating block diagrams where blocks are in parallel and in series, we get

$$
h_{a}(s)=\frac{\rho}{1+\tau s}+\frac{1}{1+\tau s}(1-\rho) h_{a}(s)
$$

Solving for $h_{a}(s)$, we get (8).

(The proof could alternativcly be undertaken by summing up an infinite series of transfer functions).

From theorem 1 follows a "look-inside corollary":

Corollary 1.1: Given a network as defined in theorem 1, and an output flow from the network, $F_{o}(t)$. Then the sum of internal flows (with)in the network is

$$
F_{w}(t)=\frac{1-\rho}{\rho} F_{o}(t)=\frac{T_{a}-\tau}{\tau} F_{o}(t)
$$

If we now allow the time lag for the network to vary, we have an "instantaneous time lag (money velocity) corollary":

Corollary 1.2: Given exogenously determined input and output flows $F_{i}(t)$ and $F_{o}(t)$. $\dot{M}(t)=-F_{o}(t)+F_{i}(t)$ is then also exogenously determined. The instantaneous time lag of the network is

$$
T_{a}(t)=\frac{M(t)}{F_{o}(t)}
$$

The instantaneous money velocity is $v_{a}=1 / T_{a}(t)$

From (13) and (12), and assumptions given below, we have a "flow depletion corollary":

Corollary 1.3: Given exogenously determined input and output flows $F_{i}(t)<F_{o}(t)$. Then $\dot{M}(t)<0$. Assume non-decreasing output flows, i.e. $F_{o}(t) \geq 0$ and some lower bound $\tau$ on the agent time lag. Then the flows within the network will decrease relative to $F_{o}(t)$,

$$
\frac{d}{d t}\left(\frac{F_{w}(t)}{F_{o}(t)}\right)<0
$$

Depletion of internal flows within a sector, as a consequence of the sector being forced to yield an exogenously decided output flow, is what happens in economy with increasing debt burdens. A model of this is derived and discussed in (Andresen, 1999).

From $T_{a}=\tau / \rho$, and (12) and (13), we have a "liquidity preference corollary":

Corollary 1.4: Assume that liquidity preference is on the increase, which in our model 
is expressed by an increasing $\tau$. It means that, all other things being equal, both aggregate output flow $F_{o}(t)$ and the aggregate of internal flows, $F_{w}(t)$, will decrease, with

$$
F_{o}(t)=\rho \frac{M(t)}{\tau(t)}, \text { and } F_{w}(t)=(1-\rho) \frac{M(t)}{\tau(t)}
$$

Such a mechanism is at work during serious economic crises, and contributes to a possible deflationary collapse followed by a depression (ibid.).

\subsection{An algebra of time lags in a network}

The reader may at this stage protest that the assumptions made until now are quite restrictive: All agents have the same first order dynamics with constant and identical time lags, all agents have the same proportion $\rho$ of spending outside its sector. We will from now on rescind the assumptions about first order dynamics, identical time constants and identical inside/outside spending proportions. The price we have to pay is that we cannot say anything about the specific response of the aggregate, only about its time lag. We can (obviously) still say that the response must be positive for all $t$, regardless of differing and time-varying dynamics for all the agents in the aggregate.

We will from now on examine agent dynamics through their impulse responses. In our continuous-time economic application, an impulse or delta function $\delta(t)$ corresponds to the agent receiving one unit of money at $t=0$. For the first order impulse response where the transfer function is given by (1), the time lag $\tau$ is also the position along the time axis of the centroid of the area under the impulse response. For an arbitrary higher order impulse response, we now define the time lag $\tau$ in the same way. If the area under the impulse response is not unity, we divide by the area,

$$
\tau=\frac{\int_{0}^{\infty} t h(t) d t}{\int_{0}^{\infty} h(t) d t} \text {, or } \tau=\int_{0}^{\infty} t h(t) d t \text { if the area is unity }
$$

If nothing else is said, we assume unit area from now on. This holds for all economic agents, since money is not created or destroyed by any such agent. We call such an impulse response PUA (positive, unit area). In the $s$ domain (Laplace transformed), a unit area impulse corresponds to unity static gain of the transfer function, we have

$$
\left.h(s)\right|_{s=0}=1
$$

Consider a PUA transfer function

$$
h(s)=\frac{n(s)}{d(s)}=\frac{1+b_{1} s+\ldots+b_{n-1} s^{n-1}}{1+a_{1} s+\ldots+a_{n-1} s^{n-1}+a_{n} s^{n}}
$$

The time lag $\tau$ may be found through the relation

$$
\tau=\int_{0}^{\infty} t h(t) d t=\left.\mathscr{L}[\operatorname{th}(t)]\right|_{s=0}=-\left.\frac{d}{d s} h(s)\right|_{s=0}
$$

We write $\tau=-h^{\prime}(0)$ for convenience. (20) applied to (19), gives

$$
\tau=\frac{n(0) d^{\prime}(0)-d(0) n^{\prime}(0)}{d^{2}(0)}=\frac{d^{\prime}(0)-n^{\prime}(0)}{1}=a_{1}-b_{1}
$$


For the special case of the first order transfer function, this confirms that $\tau$ is identical to the time constant, as already stated. For higher-order transfer functions we note that $\tau$ is independent of the coefficients $b_{i}$ and $a_{i}, i>1$.

For $N$ PUA transfer functions of the type (19) in a series connection, the resulting transfer function will also be PUA. (20) gives

$$
\begin{aligned}
\tau & =-h^{\prime}(0)=-\left(h_{1} h_{2} \ldots h_{N}\right)^{\prime}(0) \\
& =-\left(h_{1}{ }^{\prime}(0)+h_{2}{ }^{\prime}(0)+\ldots+h_{N}(0)\right)=\tau_{1}+\tau_{2}+\ldots \tau_{N}
\end{aligned}
$$

This is reasonable, considering that $\tau$ may be seen as the lag of a flow which is transmitted through a chain of $N$ "agents" (referring to our economic application). The impulse response of a series connection of sub-systems as in (22), corresponds to convolution of PUA impulse responses in the time domain. A PUA response is mathematically similar to a probability distribution function, which also is PUA. From probability theory we know that if we convolve $N$ p.d.f.s, the result is the p.d.f. for the sum of the respective random variables (assuming they are independent) (see for instance Casella and Berger, 1990, p. 210). And the Central Limit Theorem (ibid., pp. 216-218) tells us that the resulting p.d.f. will tend to a normal distribution when $N$ is large. If we return to the time domain and apply this to a system consisting of a large number of $N$ serially connected PUA subsystems, this means that the impulse response of the system will have a shape approaching that of the normal distribution, with time lag corresponding to its mean value. To explore this, consider a transfer function

$$
h_{N}(s)=\frac{1}{\left(1+\frac{\tau}{N} s\right)^{N}}
$$

It corresponds to a chain of $N$ serially connected identical first order time lags. Since each lag in the chain is $\tau / N$, rule (22) tells us that the aggregate lag will be invariant $=\tau$.

Incidentally, we note that

$$
\lim _{N \rightarrow \infty} h_{N}(s)=\mathrm{e}^{-\tau s}
$$

so that the impulse response in the limit is trivially an impulse delayed by $\tau$. We want, however, to examine the responses for large but finite $N$. The impulse response corresponding to (23) is

$$
h_{N}(t)=\frac{1}{(N-1) !}\left(\frac{N}{\tau}\right)^{N} t^{N-1} \mathrm{e}^{-\frac{N t}{\tau}}
$$

Figure 7 shows a selection of responses, with $N=1, \ldots, 30$ and $\tau=1$. (The corresponding normal distribution shape is indicated with a dotted line.)

Note that these responses are all from transfer functions with no zeroes. We will introduce a zero and use $N=2$ for Monte Carlo simulations of an economic network further below. It will be demonstrated that a zero and two poles are sufficient to generate a wide range of realistic responses.

Now to the case of PUA transfer functions connected in parallel:

$$
h=\rho_{1} h_{1}+\rho_{2} h_{2}+\ldots+\rho_{N} h_{N}
$$

Here we require $\rho_{1}+\rho_{2}+\ldots+\rho_{N}=1$ so that the unit area condition is not violated. The time lag is then a weighted average, 


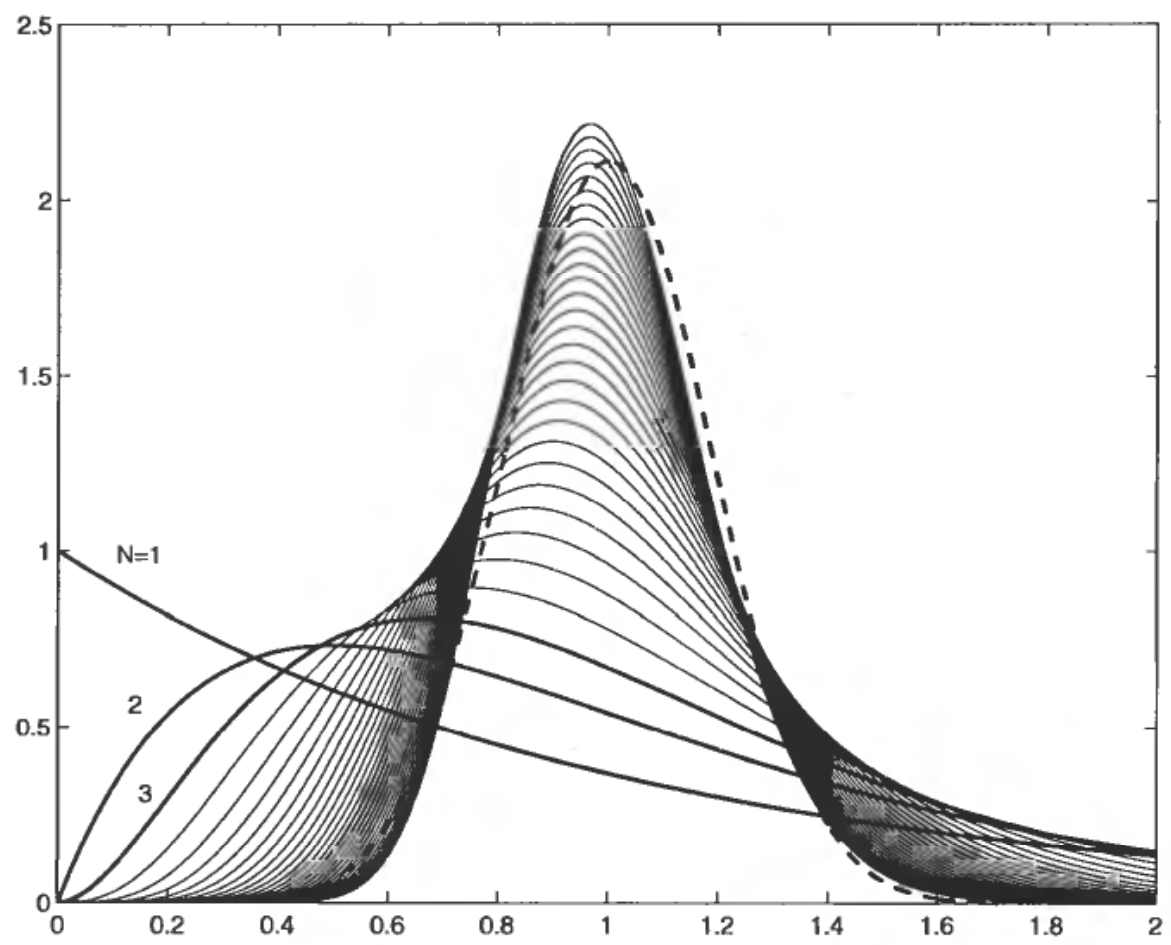

Figure 7. The impulse response of a chain of $\mathrm{N}$ transfer functions (23).

$$
\begin{aligned}
\tau & =-h^{\prime}(0)=-\left(\rho_{1} h_{1}+\rho_{2} h_{2}+\ldots+\rho_{N} h_{N}\right)^{\prime}(0) \\
& =\rho_{1} \tau_{1}+\rho_{2} \tau_{2}+\ldots+\rho_{N} \tau_{N}
\end{aligned}
$$

The series (22) and parallel (27) rules for calculating time lags might be employed to check the time lag of a network of more arbitrary transfer functions. Assume that transfer functions of the type (19) differ from agent to agent, but with similar coefficients $\tau=a_{1}$ and a common outside spending coefficient $\rho$. Consider Figure 6, but now with these more arbitrary transfer functions in the blocks. We apply rules (22) and (27), and get an equation for the time $\operatorname{lag} T_{a}$ for the aggregate by employing a similar argument as that which led to (11):

$$
T_{a}=\tau+\left(\rho \cdot 0+(1-\rho) T_{a}\right)
$$

This gives $T_{a}=\tau / \rho$, as expected.

Finally, consider a linear monovariable system on state space form with a positive-not necessarily unit area-impulse response:

$$
\begin{aligned}
& \dot{\boldsymbol{x}}=A \boldsymbol{x}+\boldsymbol{b} u \\
& y=c^{T} x
\end{aligned}
$$

The transfer function is

$$
h(s)=c^{T}(s I-A)^{-1} b
$$

The area under the response is

$$
\left.h(s)\right|_{s=0}=h(0)=-c^{T} A^{-1} b
$$


We also have

$\left.-\left.\frac{d}{d s} h(s)\right|_{s=0}=-h^{\prime}(0)=-c^{T}\left\{I-(s I-A)^{-1} I(s I-A)^{-1}\right]_{s=0}\right\} b=c^{T} A^{-2} b$

(31) and (32) in (17) then give the time lag for the system,

$$
\tau=\frac{\int_{0}^{\infty} t h(t) d t}{\int_{0}^{\infty} h(t) d t}=\frac{-h^{\prime}(0)}{h(0)}=-\frac{c^{T} A^{-2} b}{c^{T} A^{-1} b}
$$

\section{Monte Carlo simulations}

We propose that a second order transfer function with one zero and real poles is sufficient for furnishing the necessary variability in individual agent dynamics. We now express the agent PUA transfer function in the form

$$
h(s)=\frac{\alpha_{1}+\alpha_{1} T_{2} s}{\alpha_{1}+\alpha_{2} s+s^{2}}
$$

The point of the zero $-1 / T_{z}$ is to account for some agents spending a certain share of incoming money immediately after receipt. For a large $T_{z}$, the agent's spending reaction will start with a fairly strong initial pulse followed by a correspondingly small exponential tail. Figure 8 shows a collection of spending impulse responses for 10 agents, where all have a transfer function of the type (34). They are all PUA and they have the same time lag, here $\tau=1$. Each response corresponds to a specific parameter set $T_{z}, T_{p 1}, T_{p 2}$ where $-1 / T_{p 1},-1 / T_{p 2}$ are the poles of (34). Each set is generated by uniform probability density functions, through the following procedure: First a $T_{z}$ is generated in the range $0<T_{z}<0.5 \tau$ (the factor 0.5 is fairly arbitrary). According to (21) and (22), the sum of the denominator time constants must then be $\tau+T_{i}$. The next step is generating the pair $T_{p 1}, T_{p 2}$ by a uniform p.d.f., but scaled afterwards such that this condition is satisfied.

The same set of responses is shown in both windows, but with different scaling. In the lower left window we note the large initial amplitude of some responses; these are the cases with a fairly large $T_{z}$. The first-order time lag response is shown with a thick dotted line for comparison, and its tangent (and thus $\tau$ ) is also indicated. We observe that the first order time lag is not a good approximation to most of the responses that are generated.

Our conjecture, however, is that if we interconnect a large number of agents with such differing responses as shown above, we will observe that the first order time lag as an approximation improves with the number of agents. To explore this proposition, a state space model is defined. It consists of subsystems of the type (34), interconnected such that the total system is still PUA. Let

$$
\begin{aligned}
& x=A x+b u \\
& y=c^{T} x
\end{aligned}
$$

The matrix $A$ is

$$
A=\left[\begin{array}{ccccccc}
0 & 1 & 0 & 0 & 0 & 0 & \ldots \\
-\alpha_{11} & -\alpha_{12} & r_{21} \alpha_{21} & r_{21} \alpha_{21} T_{2,2} & r_{31} \alpha_{31} & r_{31} \alpha_{31} T_{z, 3} & \ldots \\
0 & 0 & 0 & 1 & 0 & 0 & \ldots \\
r_{12} \alpha_{11} & r_{12} \alpha_{11} T_{z, 1} & -\alpha_{21} & -\alpha_{22} & r_{32} \alpha_{31} & r_{32} \alpha_{31} T_{z, 3} & \ldots \\
\cdots & \cdots & \ldots & \ldots & \ldots & \ldots & \ldots
\end{array}\right]
$$




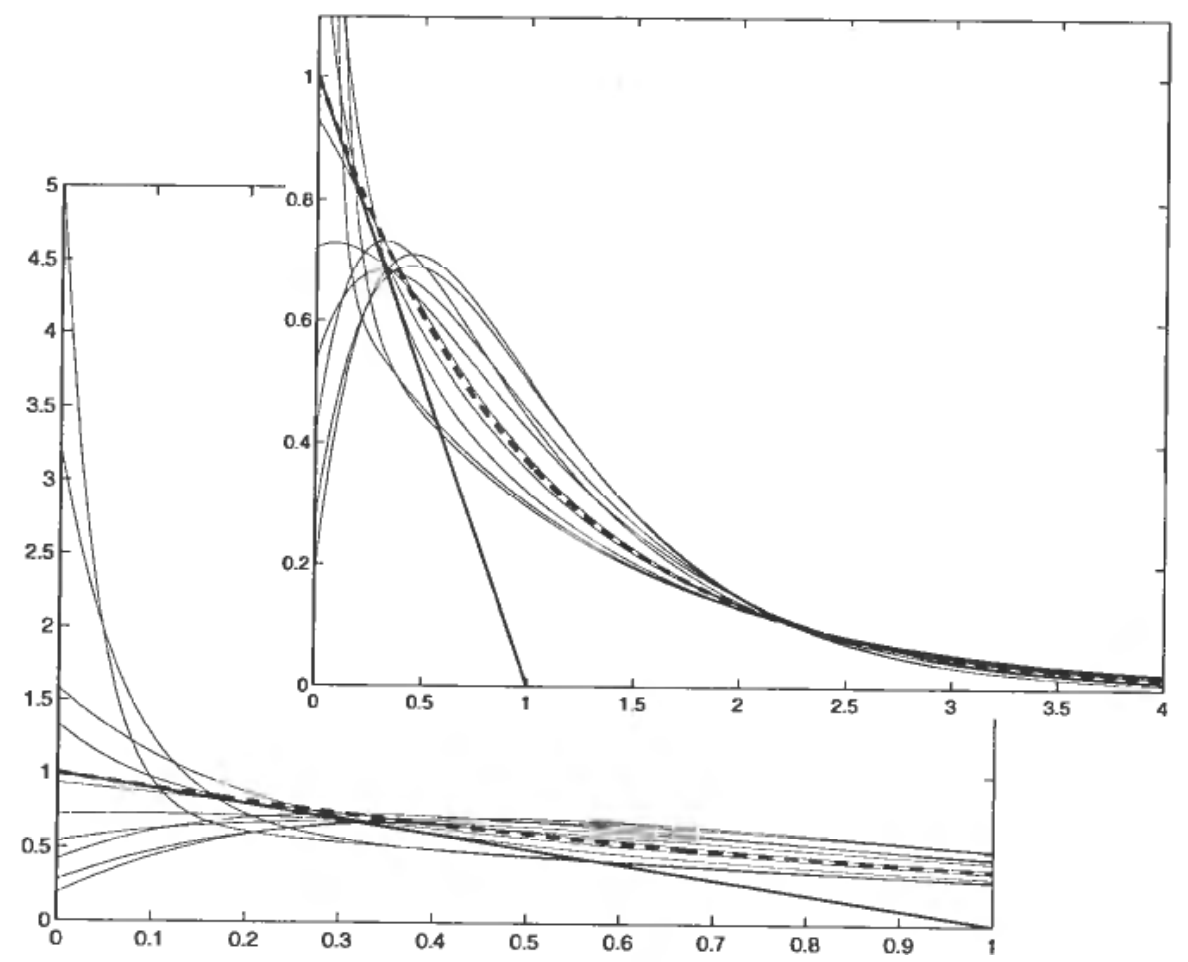

Figure 8. Ten impulse responses of (34), with a common time lag $=1$.

With $n$ subsystems we have a system dimension $2 n$. Each subsystem has transfer function (34), and is realized as a controllable canonical form (see for instance Belangér, 1995, pp. 100-104). The subsystems (agents) are indexed $i=1, \ldots, n$. The definitions for $\alpha_{i j}, T_{z, i}$ correspond to (34). Note that indices in (36) are not matrix element indices in the conventional sense, since they pertain to the subsystems. Each coefficient $r_{i k}$ accounts for the flow from subsystem $i$ to subsystem $k$. For each subsystem $i$ there is an outside spending coefficient $\rho_{i}$. Then $r_{i k}$ must satisfy

$$
\sum_{k=1, k \neq i}^{n} r_{i k}=1-\rho_{i}
$$

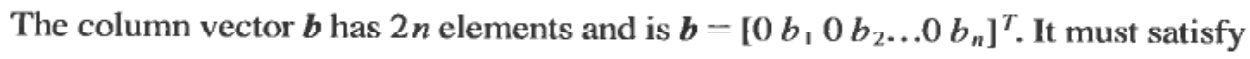

$$
\sum_{i=1}^{n} b_{i}=1
$$

Conditions (37) and (38) are necessary to achieve unit area impulse response for the total system, i.e. such that no money is created or destroyed within it.

The row vector $\boldsymbol{c}^{T}$ has $2 n$ elements and is

$$
c^{T}=\left[\rho_{1} \alpha_{11} \rho_{1} \alpha_{11} T_{z, 1} \rho_{2} \alpha_{21} \rho_{2} \alpha_{21} T_{z, 2} \ldots \rho_{n} \alpha_{n 1} T_{z, n}\right] \text {, with all } \rho_{i}<1
$$

Now to the procedure for assigning values to the above parameters: For each Monte Carlo run, a complete new set is generated. All probability distributions employed are 


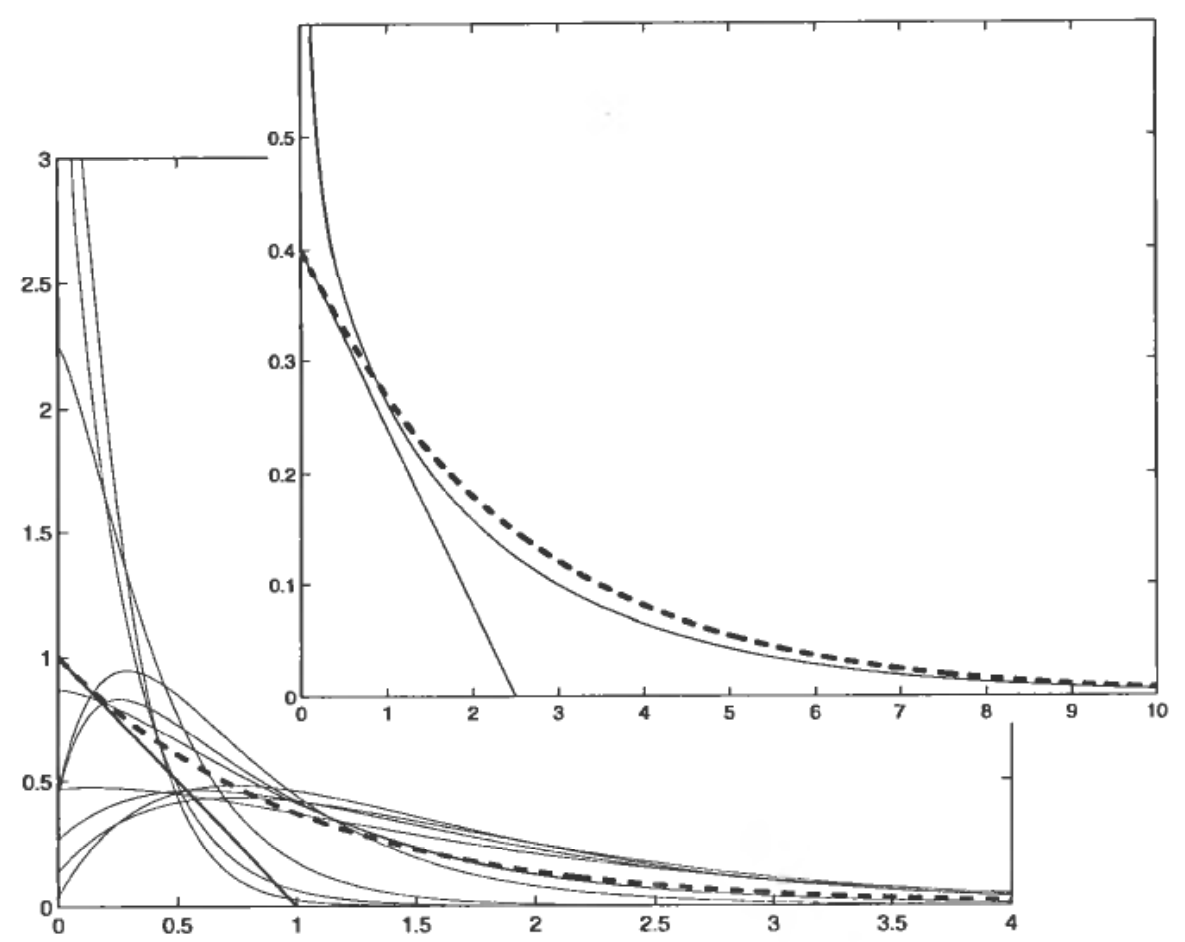

Figure 9. Ten individual responses (LL), and the corresponding aggregate response (UR).

uniform. The procedure is executed for each subsystem $i$ : First, a lag $\tau_{i}$ is drawn. Based on this, the parameters $\alpha_{i j}, T_{z, i}$ are generated as already described. Then an outside spending coefficient $\rho_{i}<1$ is drawn. Next, coefficients $r_{i k}$ are also drawn, but scaled afterwards such that condition (37) is satisfied. By this, we also have the next two elements in $\boldsymbol{c}^{T}$. After repeating this for all subsystems, all elements in $\boldsymbol{b}$ are drawn and then scaled such that (38) is satisfied.

One should expect widely differing responses, since all parameters are allowed to vary quite independently, and the distributions employed are chosen to have a fairly wide range. This makes the test of our conjecture more severe. The ranges chosen are:

$$
0.1<\tau_{i}<1.9,0.0<T_{z, i}<0.5 \tau_{i}, 0.1<\rho_{i}<0.7
$$

The mean time lag is $\mathbf{1 . 0}$ and the mean outside spending coefficient is 0.4 . Based on this, the approximative first order time lag response for the total system is predicted to be

$$
h(t)=0.4 \mathrm{e}^{-0.4 t}
$$

We start simulations with a system of only 10 agents.

The lower left window in Figure 9 shows the responses for each individual agent. We note that the responses, as opposed to those in Figure 8, are much more dispersed now since also the time lags differ between them (in the range 0.1 to 1.9). The mean first order response and its tangent is also indicated. The upper right window shows the impulse response of the "sector" consisting of these ten agents, together with the first 


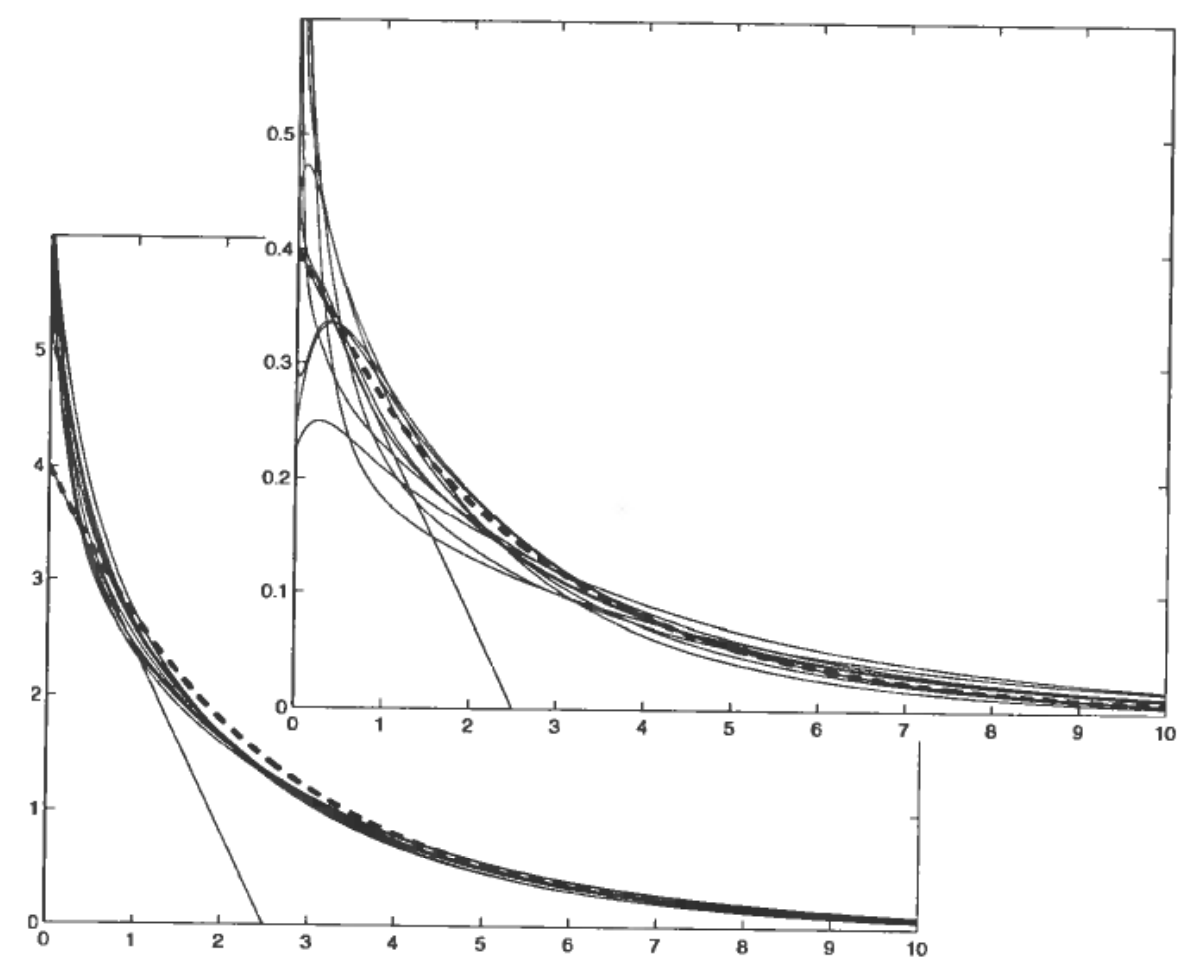

Figure 10. Aggregate responses for a 150-agenmt system (LL), and a 10-agent system (UR).

order time lag response (41). We observe that this proposed approximation is not too bad, as predicted.

A population of as little as ten agents in a sector is quite unrealistic. We therefore do the same with a 150 -agent system, which means a $300 \times 300$ system matrix. This is a fairly heavy computing task, so experiments are not carried out for higher numbers of agents. In Figure 10 are given 10 responses for a 150-agent system, in the lower left window. For comparison, 10 responses for a 10-agent system are also given, in the upper right window.

We observe that the first order approximation is better for the sector with the higher agent population. But the approximation cannot fully represent the strong spread in individual agent dynamics, with lags from 0.1 to 1.9. This explains the steep part of the true system response at its start, which is due to outputs from the agents with the fastest dynamics).

Now to the impact of the outside spending factor, until now chosen in the range $0.1<\rho_{i}<0.7$. If instead all $\rho_{i}$ were close to unity, this would mean that agents do not interact, but spend most of their money directly out of their own sector, like in the household case. In this case the sector response is simply a weighted mean of individual responses. On the other hand, if all $\rho_{i}$ are «1, this means that a unit of money in an average sense has to pass many agents before it is spent out of the sector.

We have simulated such a case, with $0.05<\rho_{i}<0.15$, i.e. a mean $\rho$ of 0.1 . Figure 11 shows 10 Monte Carlo responses for a 150 agent system.

In this case the time lag is 10 for the aggregated system, in accordance with (8). Compared to Figure 10, lower left window, we see that 


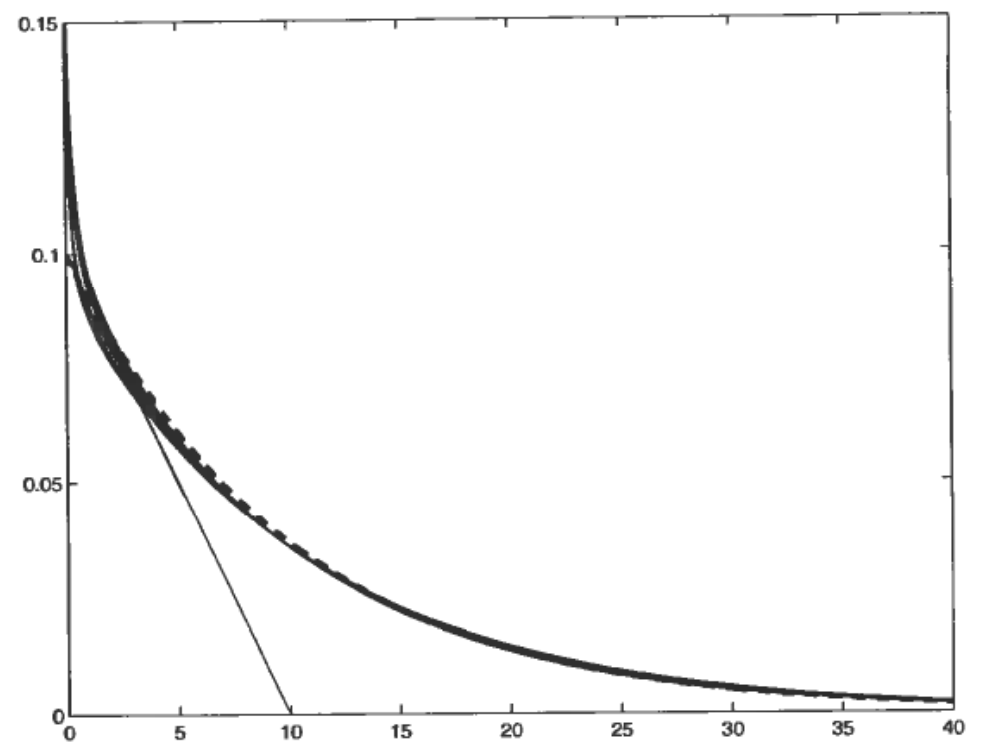

Figure 11. Aggregate responses for a 50-agent system with a low $\rho$.

- the responses are closer to the proposed first-order approximation.

- the initial spikes which are due to zeroes in transfer functions for individual agents in the network, still leave a mark on the aggregate response in the form of a corresponding initial peak, but a smaller and narrower one.

We will now try to explain this by exploiting an intermediate result from the proof given for Theorem 1. We again make the simplifying assumption that all agent transfer functions and all outside spending coefficients $\rho$ are identical. But now the agent transfer functions are of the type (34), which we here write as

$$
h(s)=\frac{1+T_{z} s}{1+a_{l} s+a_{2} s^{2}}=\frac{d(s)}{n(s)}
$$

The intermediate result (11) may be generalized to

$$
h_{a}=\frac{\rho d}{n}+\frac{d}{n}(1-\rho) h_{a},
$$

where dependence on $s$ is omitted for brevity. Solving for $h_{a}$ and using (42), we get

$$
h_{a}(s)=\frac{1+\rho T_{z}\left(\frac{s}{\rho}\right)}{1+\left(a_{1}-T_{z}[1-\rho]\right)\left(\frac{s}{\rho}\right)+\rho a_{2}\left(\frac{s}{\rho}\right)^{2}}
$$

Wc compare this to the first-order approximation, which, following (8) and (21), is

$$
h_{a}(s)=\frac{1}{1+\left(a_{1}-T_{z}\right)\left(\frac{s}{\rho}\right)}
$$


Eq. (44) confirms that the zero will makc itself felt also for the aggregate system, as already observed through the Monte Carlo runs shown in Figure 11. But (44) also tells us that its relative influence on system dynamics is less when $\rho$ is small, which is supported by a comparison of Figures 10 and 11. Furthermore, when $\rho$ is reduced, the influence on system dynamics of the second-order term in the denominator in (44) decreases in relation to the first-order term. This also supports the observation that the system response is closer to that of the first-order approximation (45), when $\rho$ is small.

\section{Conclusion}

The first order time lag approximation for a sector is vindicated. It gets better for large number of agents (which is what we have in macroeconomic applications), and still better for sectors with strong interaction (for instance firms, as opposed to households).

The model may be applied to any type of sector, and any (reasonable) size sector, and these aggregates may be interconnected.

Since agents' spending preferences are constant in each simulation, behavioural assumptions have been quite restrictive. But the model can easily incorporate time-varying coefficients, or more realistically: coefficients dependent upon system states. In the last case the model will become non-linear. This is a topic for further research.

\section{REFERENCES}

ANDRESEN, T. (1999). The dynamics of long-range financial accumulation and crisis. Forthcoming, in: Nonlinear Dynamics, Psychology, and Life Sciences, 3, No. 2.

BÉLANGER, P. R. (1995). Control engineering: a modern approach. Florida: Saunders College

Publishing, Harcourt Brace and Company.

Casella, G. and Berger, R. L. (1990). Statistical inference. California: Wadsworth, Inc.

GODLEY, W. and CRIPPS, F. (1983). Macroeconomics. Oxford University Press.

PhILlups, A. W. (1954). Stabilisation policy in a closed economy. The Economic Journal, LXIV. Phillips, A. W. (1957). Stabilisation policy and the time forms of lagged responses. The Economic Journal, LXVII. 